\title{
miR-15b promotes epithelial-mesenchymal transition by inhibiting SMURF2 in pancreatic cancer
}

\author{
WEN-LONG ZHANG ${ }^{1 *}$, JIAN-HUA ZHANG ${ }^{1 *}$, XI-ZHAO WU ${ }^{3}$, TAO YAN ${ }^{2}$ and WEI LV ${ }^{2}$ \\ Departments of ${ }^{1}$ General Surgery, ${ }^{2}$ Hepatobiliary Surgery and ${ }^{3}$ Pathology, \\ The Second Artillery General Hospital of PLA, Beijing 100088, P.R. China
}

Received April 30, 2015; Accepted June 5, 2015

DOI: 10.3892/ijo.2015.3076

\begin{abstract}
The epithelial-mesenchymal transition (EMT) is a key developmental program that is often activated during cancer invasion and metastasis. We report here that silencing SMURF2 (SMAD specific E3 ubiquitin protein ligase 2) promoted EMT in HPDE6c7 normal pancreas cells and overexpression of SMURF2 inhibited TGF- $\beta$-mediated EMT in the cells. Subsequent studies showed that SMURF2 was downregulated in pancreatic cancer tissues and it promoted mesenchymal-epithelial transition (MET) in pancreatic cancer cells as well as its expression negatively associated with gemcitabine-resistance, but it did not alter cell viability, cell cycle and cell senescence. In addition, we demonstrated that miR-15b degraded SMURF2 and its overexpression promoted EMT in pancreatic cancer, and its expression was associated with metastasis in the disease. Elucidating molecular mechanism of EMT in pancreatic cancer not only will help us to further understand the pathogenesis and progression of the disease, but also offers new targets for effective therapies.
\end{abstract}

\section{Introduction}

The epithelial-mesenchymal transition (EMT) and the reverse process, termed the mesenchymal-epithelial transition (MET), play central roles in embryogenesis (1-3). For example, for the period of early embryonic development, the mesoderm generated by EMTs develops into multiple tissue types, as well as later in development, mesodermal cells generate epithelial

Correspondence to: Dr Wei Lv, Department of Hepatobiliary Surgery, The Second Artillery General Hospital of PLA, Beijing 100088, P.R. China

E-mail:wei.lv999@yahoo.com

*Contributed equally

Abbrevations: EMT, epithelial-mesenchymal transition; MET, mesenchymal-epithelial transition; SMURF2, SMAD specific E3 ubiquitin protein ligase 2

Key words: miR-15b, SMURF2, epithelial-mesenchymal transition, pancreatic cancer organs, such as the kidney and ovary, by MET (4). Epithelial to mesenchymal transition (EMT) is an essential process for driving plasticity during development, yet it is an unintentional behavior of cells during progression of the malignant tumor (5-7). In recent years, many embryonic genes have been found to confer malignant traits, such as motility, invasiveness, and resistance to apoptosis, on neoplastic cells (8-16). The EMT-associated reprogramming of pancreatic cancer cells not only implies that fundamental changes may occur to several regulatory networks but also that an intimate interplay exists between them. Disturbance of a controlled epithelial balance is triggered by altering several layers of regulation, including the transcriptional and translational machinery, expression of non-coding RNAs, alternative splicing and protein stability (17-19).

SMURF2, a HECT-family ubiquitin ligase (E3), has been implicated in diverse biological functions including TGF- $\beta$ signaling, mitotic regulation, cell polarity, motility and chromatin modifications (20). It appears to play complex roles in tumorigenesis. Esophageal squamous cell carcinomas expressed high levels of SMURF2, which correlated with poor prognosis (21). Another study on lung adenocarcinomas and head and neck carcinomas showed a positive correlation between SMURF2 and EGFR protein levels (22). Yet, there are several reports demonstrating decreased expression of SMURF2 in other types of cancer. Protein levels of SMURF2 were found to be downregulated in human lymphoma and breast cancer tissues relative to non-cancer tissues (23) and its SMURF2 levels were lower in advanced tumors compared to less advanced organ-confined tumors, suggesting association of SMURF2 downregulation with tumor progression (24). Importantly, two recent studies using SMURF2-null mice showed that SMURF2-deficiency increased susceptibility to spontaneous tumorigenesis in various tissues including the liver, lung, pituitary and mammary gland $(23,25)$. The activity of SMURF2 to ubiquitinate and degrade RNF20, a RING-family E3 that controls histone $\mathrm{H} 2 \mathrm{~B}$ ubiquitination and genome stability, has been implicated for the tumor suppressive role of SMURF2 (23).

In this study, we found that the silencing of SMURF2 promoted EMT in HPDE6c7 normal pancreas cells and overexpression of SMURF2 inhibits TGF- $\beta$-mediated EMT in the cells. Subsequent studies showed that SMURF2 was downregulated in pancreatic cancer tissues and it promoted 
MET in pancreatic cancer cells, the expression was negatively associated with gemcitabine-resistance, but it did not alter cell viability, cell cycle and cell senescence. In addition, we demonstrated that miR-15b was able to degrade SMURF2 and its overexpression promoted EMT in pancreatic cancer and its expression was associated with metastasis in pancreatic cancer.

\section{Materials and methods}

Pancreatic cancer tissues. Nineteen patients diagnosed with pancreatic cancer were recruited from the Second Artillery General Hospital of PLA, Tongji Hospital and Hubei Cancer Center. The use of human tissue samples followed the internationally recognised guidelines as well as local and national regulations. Informed consent was obtained from each individual. The local Medical ethics committee approved the experiments undertaken. Human normal pancreas HPDE6c7, pancreatic cancer gemcitabine-sensitive cell lines HPAC, BxPC-3, Colo357, and L3.6pl (26), gemcitabine-resistant cell lines ASPC-1, Panc-1 and MiaPaCa-2 (26) were kindly donated by Dr Hua Wei (University of Michigan Medical Center, MI, USA). Briefly, cells were maintained in RPMI-1640 medium supplemented with 10\% fetal bovine serum (FBS) (Gibco, Grand Island, NY, USA) and penicillin/streptomycin at $37^{\circ} \mathrm{C}$ in a humidified atmosphere with $5 \% \mathrm{CO}_{2}$.

Plasmids, pre-miR-15b/control miR, anti-miR-15b/scramble and transfection. SMURF2-expressing plasmids/empty vectors (pcDNA3.1) were purchased from Tiangene, Tianjin, China. The expressing plasmids or empty vector (pcDNA3.1) used for each transfection is $10 \mu \mathrm{g}$. Pre-miR-15b/control miR and anti-miR-15b/scramble were purchased from Ambion, Inc. (Ambion, Austin, TX, USA). Transfection was performed with Lipofectamine 2000 reagent (Invitrogen, CA, USA) according to the manufacturer's instructions.

Western blot analysis. Western blot analysis was performed as described before (27). Briefly, after incubation with primary antibody anti-SMURF2 (1:250; Abcam, Cambridge, MA, USA), anti-E-cadherin (1:500; Abcam), anti-N-cadherin (1:200; Abcam), anti-vimentin (1:500; Abcam), anti-SNAI1 (1:500; Abcam), anti-TGFB1 (1:800; Abcam), anti-TWIST (1:500; Abcam), anti-ZEB1 (1:500; Abcam), anti-TGFB2 (1:500; Abcam), anti- $\alpha$-SMA (1:500; Abcam) and anti- $\beta$-actin (1:500; Abcam) overnight at $4^{\circ} \mathrm{C}$, IRDye ${ }^{\mathrm{TM}}-800$ conjugated anti-rabbit secondary antibodies (Li-COR, Biosciences, Lincoln, NE, USA) were used for $30 \mathrm{~min}$ at room temperature. The specific proteins were visualized by Odyssey ${ }^{\mathrm{TM}}$ Infrared Imaging System (Gene Co., Lincoln, NE, USA).

Migration and invasion assay. For transwell migration assays, $5 \times 10^{4}$ cells were plated in the top chamber with the non-coated membrane (24-well insert; pore size, $8 \mathrm{~mm}$; BD Biosciences, San Jose, CA, USA). For invasion assays, $1.25 \times 10^{5}$ cells were plated in the top chamber with Matrigelcoated membrane (24-well insert; pore size, $8 \mathrm{~mm}$; BD Biosciences). In both assays, cells were plated in medium without serum or growth factors, and medium supplemented with serum was used as a chemoattractant in the lower chamber. The cells were incubated for $36 \mathrm{~h}$ and cells that did not migrate or invade through the pores were removed by a cotton swab. Cells on the lower surface of the membrane were stained with the Diff-Quick Staining Set (Dade) and counted.

Wound healing assay. Cells $\left(6 \times 10^{5}\right)$ were seeded onto each 35-mm glass bottom dish (MatTek Co., Ashland, MA, USA) and cultured at $37^{\circ} \mathrm{C}$ with $5 \% \mathrm{CO}_{2}$ for $36 \mathrm{~h}$. The confluent monolayer of cells was wounded. Mono-layer of cells were wounded with yellow pipette tips. After washing with PBS, the cells were incubated in fresh culture medium. The wounded areas were photographed at the beginning $(0 \mathrm{~h})$ and the end $(12,24,48$ or $72 \mathrm{~h})$ of the assay with Nikon inverted microscope (ECLIPSE TE-2000U, Nikon, Japan) equipped with a video camera (DS-U1, Nikon).

Immunofluorescence analyses. For immunofluorescence analyses, cells were plated on glass coverslips in 6-well plates and transfected as indicated. At $36 \mathrm{~h}$ after transfection, coverslips were stained with the anti-SMURF2, anti-vimentin antibody or anti-E-cadherin antibody. Alexa Fluor 488 goat anti-rabbit IgG antibody was used as secondary antibody (Invitrogen). Coverslips were counterstained with DAPI (Invitrogen-Molecular Probes, Eugene, OR, USA) for visualization of nuclei. Microscopic analysis was performed with a confocal laser-scanning microscope (Leica Microsystems, Bensheim, Germany). Fluorescence intensities were measured in several viewing areas for 300 cells per coverslip and analyzed using ImageJ $1.37 \mathrm{v}$ software (http://rsb.info.nih.gov/ ij/index.html).

Reverse-transcription polymerase chain reaction (RT-PCR) and quantitative real-time RT-PCR ( $q R T-P C R)$ for SMURF2. RT-PCR and qRT-PCR were described before (27). The primer sequences for SMURF2: forward, 5'-CGATGGCTGTTA GCAGCTTTTC-3' and reverse, 5'-TGCCTCTGCAGGGCTT CAAAG-3'. TGF $\beta 1$ : forward, 5'-CGATGGCTGTTAGCAG CTT TTC-3' and reverse, 5'-TGCCTCTGCAGGGCTTC AAAG-3'.

Luciferase reporter assay. The 3' untranslated region (3'-UTR) of human SMURF2 mRNA was cloned into pRL-TK (Promega, Madison, WI, USA) using PCR-generated fragment. Site-directed mutagenesis of the miR-15b targetsite in the SMURF2-3'-UTR was carried out using Quik change-mutagenesis kit (Stratagene, Heidelberg, Germany), with SMURF2-WT-luc as a template. For reporter assays, cells were transiently transfected with WT or mutant reporter plasmids and microRNA or anti-microRNA (as indicated in Fig. 5N-P) using Lipofectamine 2000 (Invitrogen). Reporter assays were performed $36 \mathrm{~h}$ post-transfection using the Dual-luciferaseassay-system (Promega), normalized for transfection efficiency by cotransfected Renilla-luciferase.

Real-time PCR for miRNA. Total RNA from cultured cells, with efficient recovery of small RNAs, was isolated using the mirVana miRNA Isolation kit (Ambion). Detection of the mature form of miRNAs was performed using the mirVana qRT-PCR miRNA detection kit, according to the manufac- 
A

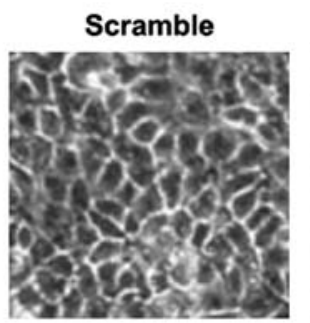

C

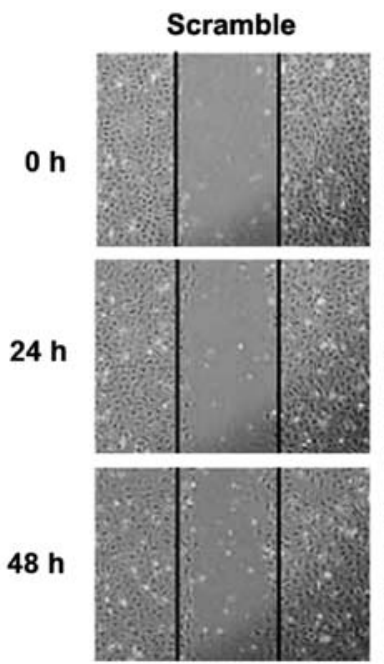

ShSMURF2

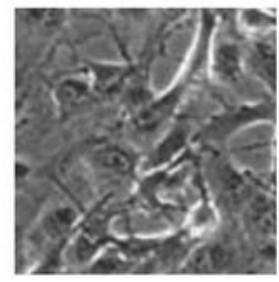

ShSMURF2
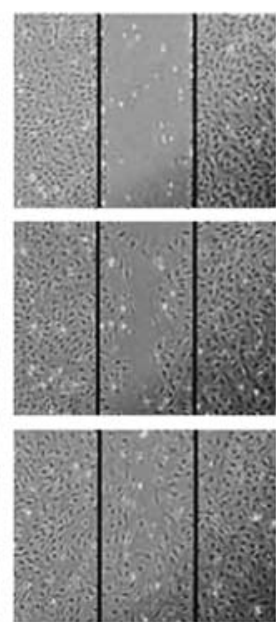

B
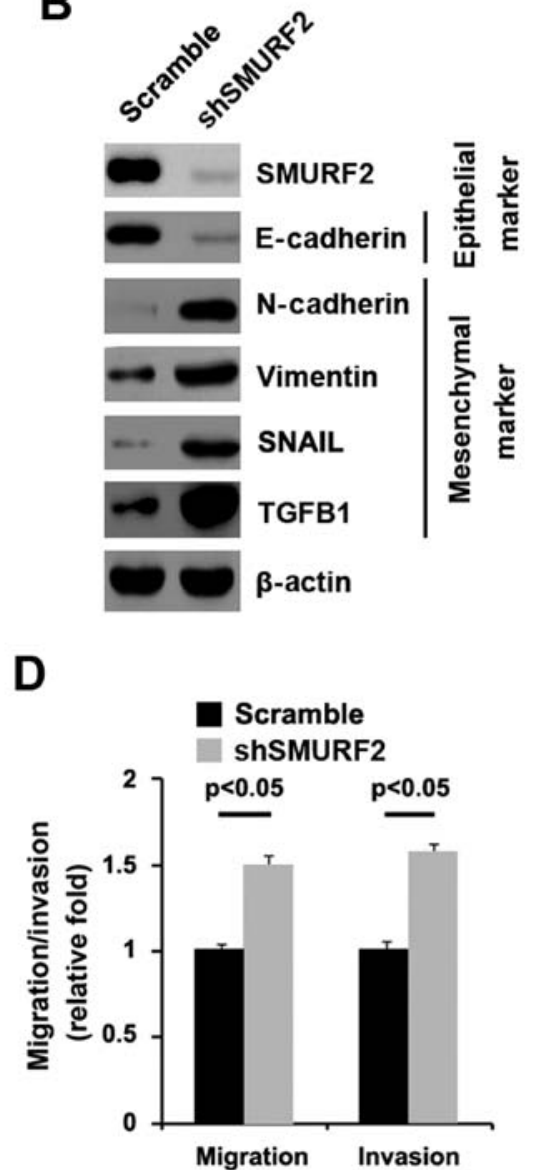

Figure 1. Silencing SMURF2 promotes EMT phenotype in HPDE6c7 normal pancreas cells. (A) HPDE6c7 normal pancreas cells were transfected as indicated. Cells were then photographed. $n=3$. (B) Western blot analysis for SMURF2, E-cadherin, N-cadherin, vimentin SNAIL and TGFB1 in HPDE6c7 cells. $\beta$-actin was a loading control. $n=3$. (C) Wound-healing assays for HPDE6c7 cells transfected as indicated. The cell layer was photographed. $n=3$. (D) Invasion and migration assays for HPDE6c7 cells transfected as indicated. $\mathrm{n}=3$.

turer's instructions (Ambion). The U6 small nuclear RNA was used as an internal control.

MTT assay. To perform the MTT assay, cells ( $0.5 \times 10^{4} /$ well) were plated in 96 -well sterile plastic plates and allowed to attach overnight. Cells were transfected as indicated. After $72 \mathrm{~h}, 15 \mathrm{ml}$ of MTT solution $(5 \mathrm{mg} / \mathrm{ml})$ was added to each well and plates were incubated for $4 \mathrm{~h}$. Crystalline formazan was solubilized with $100 \mathrm{ml}$ of a $10 \%$ (w/v) SDS solution.

Cell cycle analysis. Cells were starved for $24 \mathrm{~h}$ by deprivation of serum to synchronize the cell cycle, and then transfected as indicated. After transfection for 48, the cells were collected and fixed, and then incubated with RNase A (Invitrogen). Propidium iodide (PI) (Sigma, MO, USA) was added, followed by a $30-$ min incubation in the dark. Cellular DNA content was analyzed by a FACS (Becton-Dickinson, Franklin Lakes, NJ, USA). Data were processed using Modfit Lt software (Version SM1120, Verity Software House, USA).

Senescence-associated $\beta$-galactosidase staining. Cells seeded in 6-well plates were transfected as described previously. After $72 \mathrm{~h}$, the cells were rinsed with PBS, fixed and then incubated with freshly prepared senescence-associated $\beta$-galactosidase
(SA- $\beta$-gal) staining solution at $37^{\circ} \mathrm{C}$ overnight. A total of 200 cells were counted and percentages of SA- $\beta$-gal-positive cells calculated.

Statistical analysis. Data are presented as mean \pm SEM. Student's t-test (two-tailed) was used to compare two groups $\mathrm{P}<0.05$ was considered significant, unless otherwise indicated $\left(\chi^{2}\right.$ test $)$.

\section{Results}

Silencing SMURF2 promotes EMT phenotype in HPDE6c7 normal pancreas cells. In order to identify the role of SMURF2 in normal pancreas, we transfected HPDE6c7 cells with shSMURF2 plasmids and then western blot analysis was performed. We found that SMURF2 protein was significantly decreased in the cells transfected with shSMURF2 plasmids (Fig. 1B) and its reduction caused significant changes in HPDE6c7 cell morphology (EMT) (Fig. 1A). To further verify that the changes in cell morphology are caused by EMT, expression levels of epithelial and mesenchymal markers were compared in HPDE6c7 cells transfected with shSMURF2 plasmids with the cells transfected with scramble. The results revealed that the epithelial markers (E-cadherin) 
A

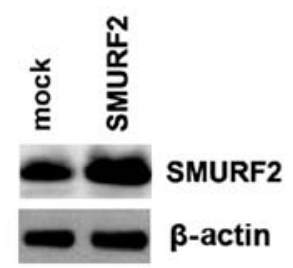

C

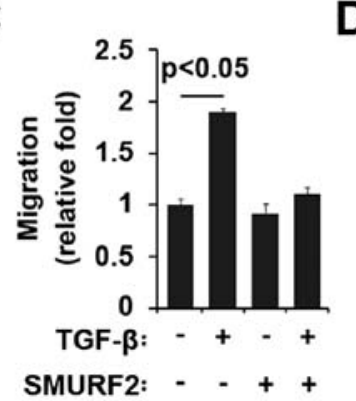

B

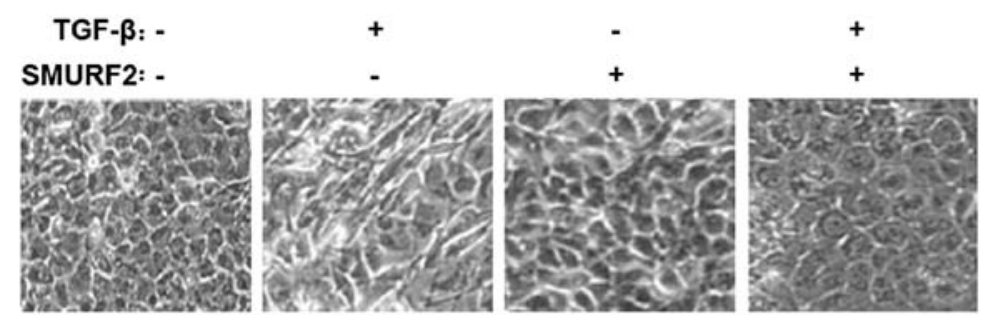

E
D

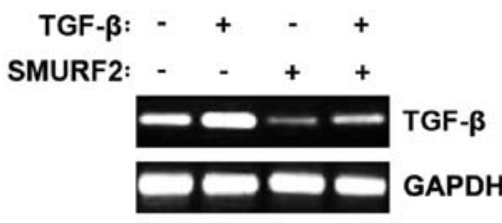

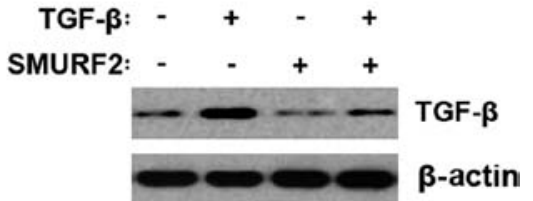

Figure 2. SMURF2 inhibits TGF- $\beta$-mediated EMT in HPDE6c7 normal pancreas cells. (A) Western blot analysis for SMURF2 in HPDE6c7 cells. $\beta$-actin was a loading control. $n=3$. (B) HPDE6c7 normal pancreas cells were treated as indicated. Cells were then photographed. $\mathrm{n}=3$. (C) Migration assays for HPDE6c7 cells transfected as indicated. $n=3$. (D) RT-PCR for TGF- $\beta$ in HPDE6c7 cells treated as indicated. GAPDH was a loading control. $n=3$. (E) Western blot analysis for TGF- $\beta$ in HPDE6c7 cells treated as indicated. $\beta$-actin was a loading control. $n=3$.

were significantly repressed, whereas mesenchymal markers (N-cadherin, vimentin, SNAIL and TGFB1) were induced by silencing SMURF2 in the cells (Fig. 1B). EMT can result in increased cell invasion and migration (28-30). Thus, we reasoned that SMURF2 could also affect invasion and migration in HPDE6c7 cells. To identify this reason, we performed would healing, invasion, and migration assay. We found that silencing SMURF2 resulted in enhanced invasion (Fig. 1D) and migration (Fig. 1C and D) in the cells.

SMURF2 inhibits TGF- $\beta$-mediated EMT. Having demonstrated that silencing SMURF2 promoted EMT phenotype in HPDE6c7 normal pancreas cells, we reasoned that SMURF2 overexpression could reverse EMT, namely promoting MET. TGF- $\beta$ can induce epithelial to mesenchymal transition (31). Thus, we reasoned that SMURF 2 could reverse TGF- $\beta$-induced EMT. We treated HPDE6c7 cells with TGF- $\beta$ for 7 days, a standard treatment that induces EMT (32) and then western blot analysis was performed. We found that TGF- $\beta$ protein was significantly increased in the cells (data not shown). We also performed western blot analysis in the cells transfected with SMURF2 expressing plasmids or empty vector. The results showed SMURF2 was significantly increased by the expressing plasmids (Fig. 2A). Similar to previous observations, TGF- $\beta$ treatment induced EMT phenotype from a cobblestone-like to a spindle-like morphology (Fig. 2B), accompanied by increase of migration in the cells (Fig. 2C). As expected, we found that SMURF2 not only reversed the change of morphology induced by TGF- $\beta$ (Fig. $2 \mathrm{~B}$ ), but also eliminated the increase of migration promoted by TGF- $\beta$ (Fig. 2C) in HPDE6c7 cells. Next, in an attempt to identify the role of SMURF2 in regulating TGF- $\beta$ expression of HPDE6c7 cells, the cells were treated as indicated. After treatment, TGF- $\beta$ mRNA expression was detected by RT-PCR and the results showed that TGF- $\beta$ mRNA was increased by TGF- $\beta$ in the cells, but the increase was attenuated by SMURF2 (Fig. 2D). We also performed western blot analysis to detect TGF- $\beta$ protein, and found that TGF- $\beta$ was also attenuated by SMURF2 (Fig. 2E). Thus, we concluded that SMURF2 could inhibit TGF- $\beta$-mediated EMT.

SMURF2 is downregulated in pancreatic cancer tissues and its expression is negatively associated with gemcitabine resistance and it promotes MET in pancreatic cancer cells. In an attempt to identify SMURF2 expression between pancreatic cancer tissues and adjacent normal tissues, we performed western blot analysis in cancer tissues versus normal tissues. Protein was isolated from 10 pairs of pancreatic cancer tissues and normal tissues (patient nos. 1-10). We found that SMURF2 protein was significantly decreased in cancer tissues (C), compared with adjacent normal tissues (N) (Fig. 3A). It implied that SMURF2 could be a tumor suppressive gene in pancreatic cancer. In an attempt to identify the SMURF2 protein expression among different pancreatic cancer cell lines, we performed western blot analysis in pancreatic cancer cell lines (HPAC, BxPC-3, Colo357, L3.6pl, ASPC-1, PANC-1 and $\mathrm{MiaPaCa}-2$ cells). Protein isolated from the 7 cell lines was detected by western blot analysis and the results showed that the expression of SMURF2 was lower in gemcitabineresistant cells (ASPC-1, PANC-1 and MiaPaCa-2 cells) than gemcitabine-sensitive cells (HPAC, BxPC-3, Colo357 and L3.6pl) (Fig. 3B).

Next, in order to identify whether EMT was associated with gemcitabine-resistance, we performed western blot analysis in gemcitabine-sensitive cells (HPAC, BxPC-3 and Colo357) and gemcitabine-resistant cells (PANC-1 and MiaPaCa-2 cells) to detect vimentin (mesenchymal marker) and E-cadherin (epithelial marker). The results of western blot analysis showed 
A

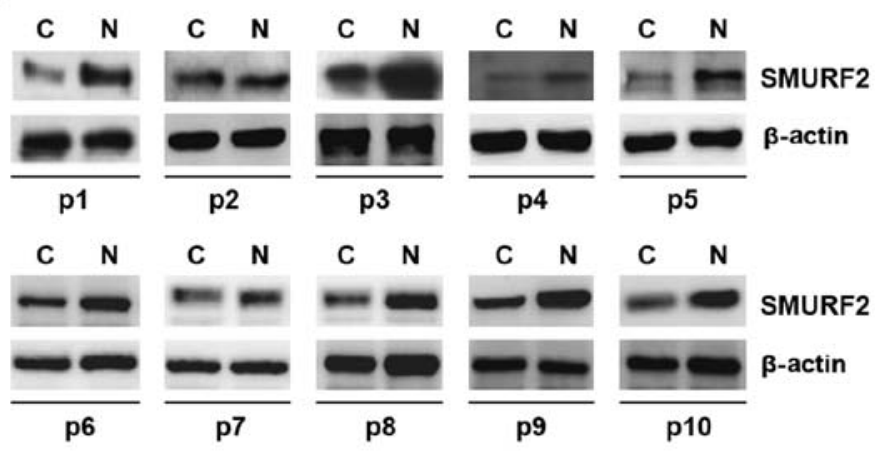

C

gemcitabine gemcitabine resistant sensitive

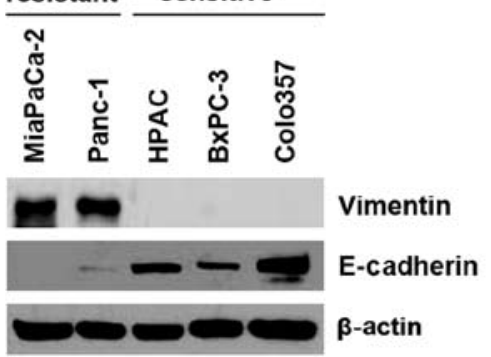

$\mathbf{F}$
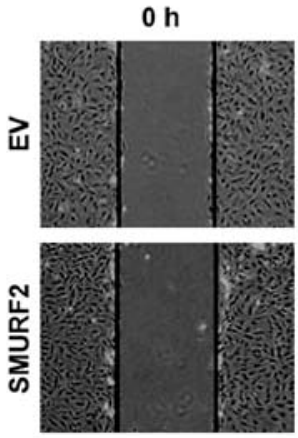

D

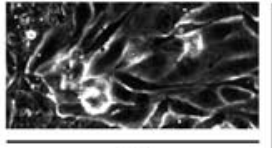

EV

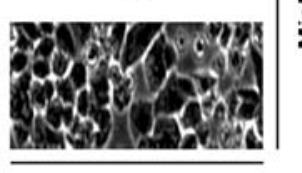

SMURF2

B

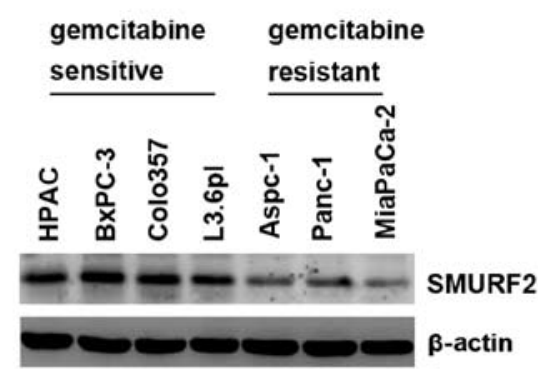

$\mathbf{E}$

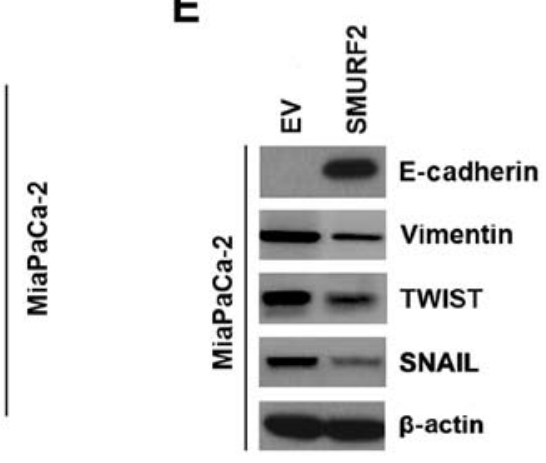

G

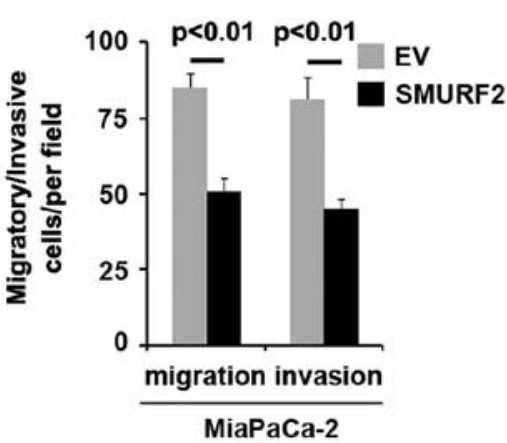

Figure 3. SMURF2 is a tumor suppressor in pancreatic cancer. (A) Western blot analysis for SMURF2 in pancreatic cancer tissues (C) and adjacent normal tissues $(\mathrm{N})$. $\beta$-actin was a loading control. $\mathrm{n}=10$. (B) Western blot analysis for SMURF2 in pancreatic cancer cell lines. $\beta$-actin was a loading control. $\mathrm{n}=3$. (C) Western blot analysis for vimentin and E-cadherin in pancreatic cancer cell lines. $\beta$-actin was a loading control. $n=3$. (D) MiaPaCa- 2 cells were transfected with empty vectors (EV) or SMURF2 expressing plasmids. Cells were then photographed. $\mathrm{n}=3$. (E) Western blot analysis for E-cadherin, vimentin, TWIST and SNAIL in pancreatic cancer MiaPaCa-2 cells. $\beta$-actin was a loading control. $n=3$. (F) Wound-healing assays for MiaPaCa-2 cells transfected with empty vectors (EV) or SMURF2 expressing plasmids. The cell layer was photographed. $n=3$. (G) Invasion and migration assays for MiaPaCa-2 cells transfected with empty vectors $(\mathrm{EV})$ or SMURF2 plasmids. $\mathrm{n}=3$.

that vimentin was detected only in gemcitabine-resistant cells (PANC-1 and MiaPaCa-2 cells) and E-cadherin was significantly elevated in gemcitabine-sensitive cells, compared with gemcitabine-resistant cells (Fig. 3C).

Because SMURF2 was downregulated in gemcitabineresistant pancreatic cancer cells and gemcitabine-resistant pancreatic cancer cells was associated with EMT and SMURF2 inhibited EMT in normal pancreatic cells, we reasoned that SMURF2 was associated with EMT in pancreatic cancer. In order to assess the role of SMURF2 in pancreatic cancer, we transfected MiaPaCa-2 cells with SMURF2 expressing plasmids and then western blot analysis was performed. We found that SMURF2 protein was significantly increased in the cells transfected with SMURF2 expressing plasmids (data not shown) and its overexpression caused significant changes in MiaPaCa-2 cells morphology (MET) (Fig. 3D). To further verify that the changes in cell morphology are caused by EMT, expression levels of epithelial and mesenchymal markers were compared in MiaPaCa- 2 cells transfected with SMURF2 expressing plasmids with the cells transfected with empty vectors. The results revealed that the epithelial marker (E-cadherin) was promoted, whereas mesenchymal markers (vimentin, TWIST and SNAIL) were suppressed by SMURF2 overexpression in the cells (Fig. 3E). In order to identify whether SMURF2 overexpression affected migration and invasion, we performed would healing, migration and invasion 
A

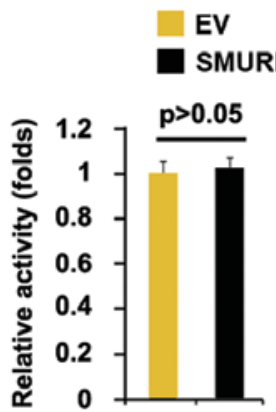

B

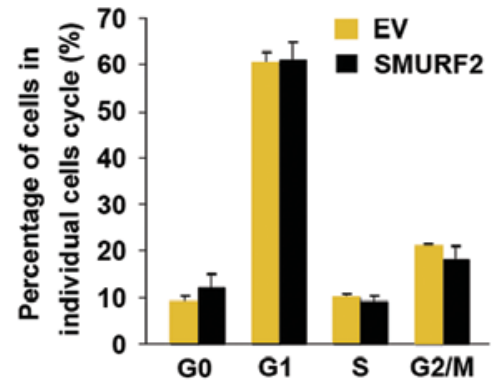

C

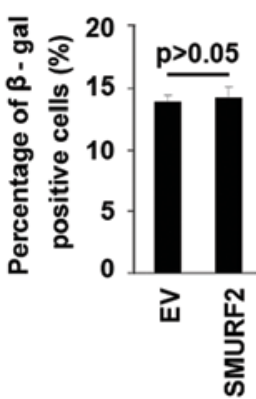

D

E

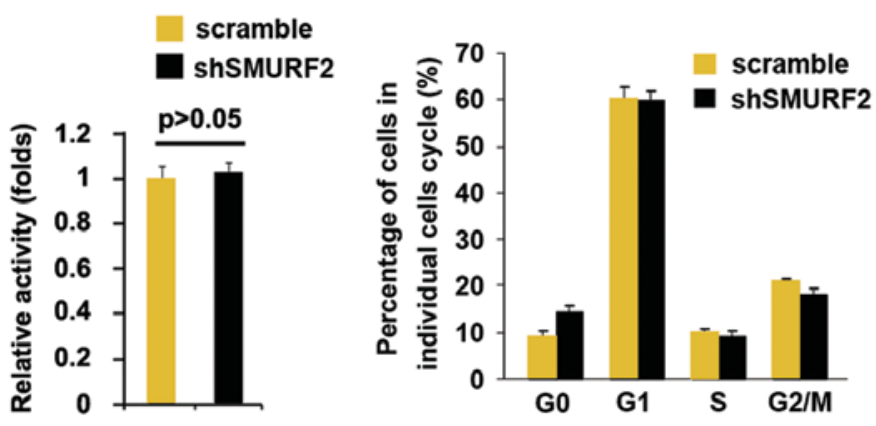

$\mathbf{F}$

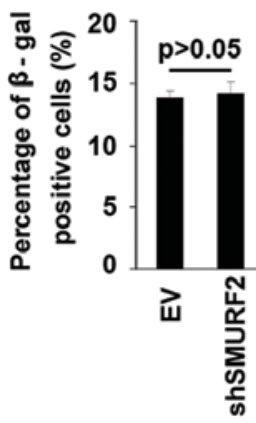

Figure 4. SMURF2 does not alter cell viability, cell cycle or senescence in pancreatic cancer. (A) MTT assay for MiaPaCa-2 cells transfected with empty vectors (EV) or SMURF2 expressing plasmids. $n=3$. (B) Cell cycle analysis for MiaPaCa-2 cells transfected with empty vectors (EV) or SMURF2 expressing plasmids. $n=3$. (C) $\beta$-gal senescence assay for MiaPaCa-2 cells transfected with empty vectors (EV) or SMURF2 expressing plasmids. $n=3$. (D) MTT assay for MiaPaCa-2 cells transfected with scramble or shSMURF2. $\mathrm{n}=3$. (E) Cell cycle analysis for MiaPaCa-2 cells transfected with scramble or shSMURF2. $\mathrm{n}=3$. (F) $\beta$-gal senescence assay for MiaPaCa- 2 cells transfected with scramble or shSMURF2. $n=3$.

assay in MiaPaCa-2 cells. The results showed that SMURF2 suppressed migration (Fig. 3F and G) and invasion (Fig. 3G) in the cells. Thus, we concluded that SMURF2 is downregulated in pancreatic cancer tissues and negatively associated with gemcitabine resistance and it promotes MET in pancreatic cancer cells.
SMURF2 does not alter cell viability, cell cycle and cell senescence. To identify whether SMURF2 could alter proliferation, we performed an MTT assay and cell cycle analysis. However, the result of MTT demonstrated that SMURF2 did not affect viability of MiaPaCa-2 (Fig. 4A) and the cell cycle was not changed by its overexpression in the cells (Fig. 4B). 
Next, we performed $\beta$-gal senescence assay, but the results showed that SMURF2 overexpression did not alter cell senescence (Fig. 4C). Having demonstrated that SMURF2 overexpression did not alter cell viability, cell cycle and cells senescence in MiaPaCa-2 cells, to provide further evidence that SMURF2 was not involved in viability, cells cycle and cell senescence of MiaPaCa-2 cells, we studied the effects of the inhibitor of SMURF2, the shSMURF2. shSMURF2 significantly downregulated SMURF2 expression in MiaPaCa-2 cells (data not shown). After stable transfection, we performed MTT assay, cell cycle analysis and $\beta$-gal senescence assay to detect cell viability, cell cycle distribution and cell senescence of MiaPaCa- 2 cells transfected with shSMURF2 plasmids and scramble. Silencing SMURF2 did not alter viability (Fig. 4D), cell cycle distribution (Fig. 4E) or cell senescence (Fig. 4F).

miR-15b degrades SMURF2 in pancreatic cancer cells. Having demonstrated that SMURF2 expression is specifically downregulated in pancreatic cancer (Fig. 3A) and it suppressed EMT in vitro, we investigated which mechanisms suppressed SMURF2 expression in pancreatic cancer. MicroRNAs (miRs) are a class of small noncoding RNAs ( 22 nucleotides) negatively regulating protein-coding gene expression by targeting mRNA degradation or translation inhibition (20,21,33). Upregulation of specific miRNA can contribute to downregulation of a tumor suppressive gene (34-36). Thus we reasoned that SMURF2 was downregulated by overexpression of specific miRNA in pancreatic cancer.

In an attempt to identify the level of miRNA expression in pancreatic cancer $\mathrm{MiaPaCa}-2$ cells (low expression of SMURF2) and BxPC-3 cells (high expression of SMURF2) (Fig. 3B), we performed miRNA profiling in the cell lines. RNAs isolated from the two cell lines were hybridized to a custom miRNA microarray platform. After hybridization, quantification, and normalization, we found that miR-15b, miR-212 and miR-155 were significantly increased in MiaPaCa-2 cells compared with BxPC-3 cells >100-fold (Fig. 5A).

As further confirmation, we used 2 commonly used prediction algorithms - TargetScan (http://www.targetscan.org) and PicTar (http://pictar.mdc-berlin.de/) to analyze 3'-UTR of SMURF2. The algorithms predicted that miR-15a/b, miR-16, miR-195 and miR-200c could target 3'-UTR of SMURF2 (Fig. 5B). However, we found that only miR-15b was negatively associated with SMURF2 expression in pancreatic cancer cells. Target sites of miR-15b on 3'-UTR of SMURF2 are showed in Fig. 5C. We reasoned that miR-15b could downregulate SMURF2 expression by targeting its 3'-UTR in pancreatic cancer and that SMURF2 was downregulated in pancreatic cancer cells due to overexpression of miR-15b. In an attempt to identify the role of miR-15b in regulating SMURF2 expression in pancreatic cancer cells, BxPC-3 cells were transfected with pre-miR-15b and control miR. After transfection, miR-15b expression was detected by real-time PCR and the results showed that miR-15b was significantly increased by pre-miR-15b in the cells (Fig. 5D). To confirm the reason, we performed western blot analyses in BxPC-3 cells transfected with pre-miR-15b or control miR. The results showed that SMURF2 protein was evidently suppressed in the cells transfected with pre-miR-15b at different time-points
(Fig. 5E). Next, we performed immunofluorescence analysis and RT-PCR to detect SMURF2 expression in BxPC-3 cells transfected with pre-miR-15b or control miR. The results showed that SMURF2 protein (Fig. 5F) and mRNA (Fig. 5G) were significantly downregulated in the cells transfected with pre-miR-15b. Consistent with the results of RT-PCR, real-time PCR demonstrated that SMURF2 mRNA was not reduced in BxPC-3 cells transfected with pre-miR-15b, compared with control miR-transfected groups (Fig. 5H).

Having demonstrated that miR-15b degrades SMURF2 in BxPC-3 cells, in order to further confirm that miR-15b degrades SMURF2 in pancreatic cancer cells, we performed western blot analysis, RT-PCR and real-time PCR to detect whether SMURF2 could be suppressed by pre-miR-15b in MiaPaCa- 2 cells. We showed that miR-15b could be significantly increased by pre-miR-15b (Fig. 5I) in the cells and SMURF2 protein (Fig. 5J) and mRNA (Fig. 5K and L) were evidently inhibited by it.

To further demonstrate the direct regulation of SMURF2 by $\mathrm{miR}-15 \mathrm{~b}$, we constructed luciferase reporters with the targeting sequences of wild-type (SMURF2-WT-luc, S-W) and mutated SMURF2 3'-UTRs (SMURF2-A-MUT-luc, S-M) (Fig. 5M). Both the wild-type and mutant reporters were introduced into BxPC-3 cells and MiaPaCa- 2 cells. We found that SMURF2-WT-luc plasmids were suppressed in MiaPaCa-2 cells (miR-15b high expression).

Next, to identify that miR-15b directly targets 3'-UTR of SMURF2, luciferase reporter assay was performed in BxPC-3 cells. Luciferase reporter assay showed that the luciferase activities of SMURF2-WT-luc plasmids were significantly suppressed in the cells transfected with pre-miR-15b, implying that miR-15b targeted 3'-UTR of SMURF2 mRNA (Fig. 5O). In order to further identify that miR-15b targeted 3'-UTR of SMURF2 by the predicted sites, we mutated 3 bases in the predicted sites (Fig. 5M). In addition, mutant reporters were introduced into BxPC-3 cells, as expected the luciferase activities of SMURF2-MUT-luc were not suppressed by miR-15b in BxPC-3 cells (Fig. 5O).

We also performed luciferase reporters assay in $\mathrm{MiaPaCa}-2$ cells. First, we found that anti-miR-15b could significantly downregulate miR-15b expression in MiaPaCa-2 cells (data not shown) and then luciferase reporters assay showed that the luciferase activities of SMURF2-WT-luc plasmids were significantly promoted in the cells transfected with anti-miR-15b, implying that miR-15b targeted 3'-UTR of SMURF2 mRNA (Fig. 5P). In order to further identify that miR-15b targeted 3'-UTR of SMURF2 by the predicted sites in MiaPaCa-2 cells, mutant reporters were introduced into $\mathrm{MiaPaCa}-2$ cells, as expected the luciferase activities of SMURF2-MUT-luc were not promoted by silencing miR-15b in MiaPaCa- 2 cells (Fig. 5P).

miR-15b overexpression promotes EMT in pancreatic cancer. We had demonstrated that SMURF2 was downregulated in pancreatic cancer tissues and it promoted MET in pancreatic cancer cells and miR-15b degraded SMURF2 in pancreatic cancer cells. Moreover, miR-15b was upregulated in pancreatic cancer, implying that it is an oncogene (37). Thus, we reasoned that contrary to SMURF2, miR-15b might promote EMT in pancreatic cancer. miR-15b could be increased 
A

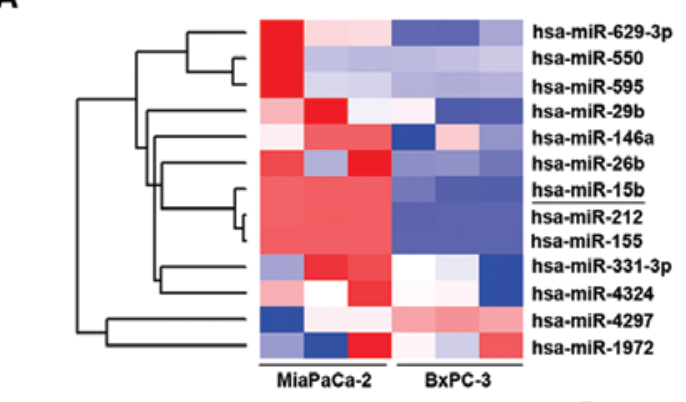

C

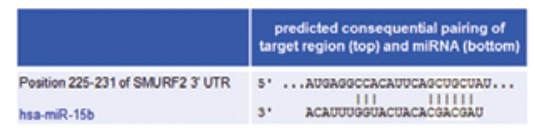

$\mathbf{F}$

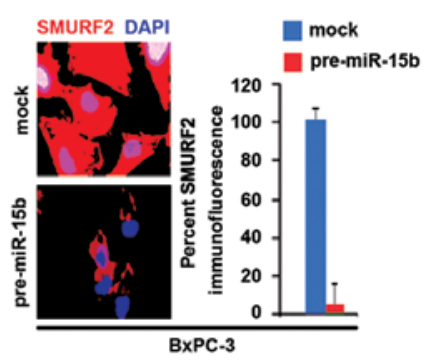

J

$\mathbf{K}$

G
D
B

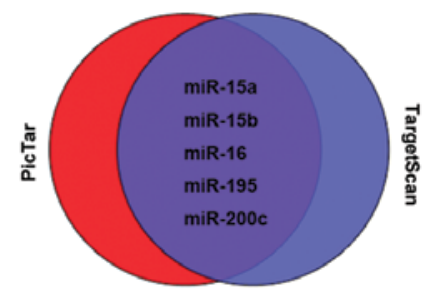

E

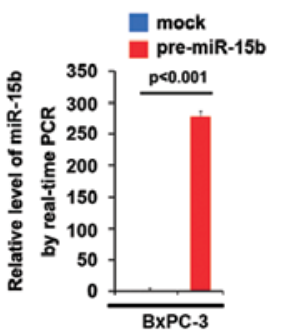

H

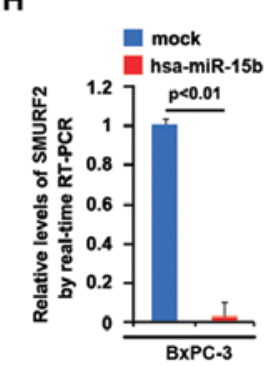

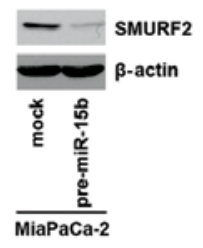

N

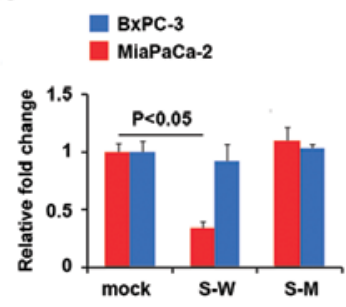

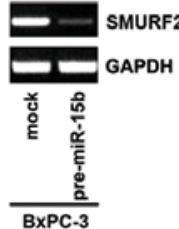

L

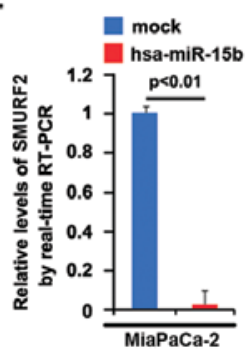

o

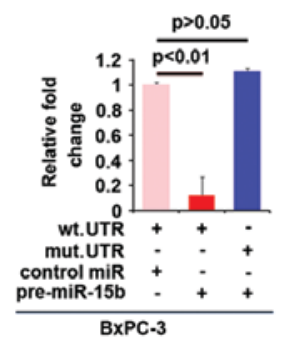

I

M
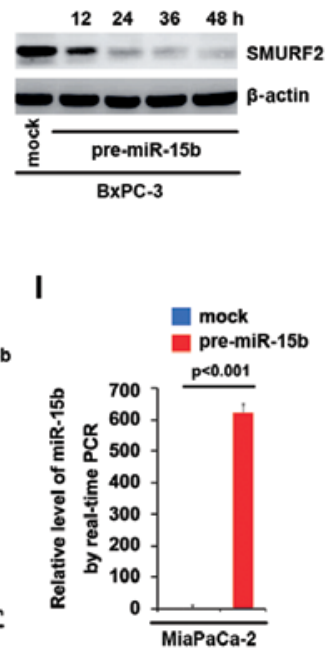

$$
\text { Position 225-231 of }
$$

SMURF2 3'UTR

5. mutation

5'...AUGAGGCCACAUUCAGCUAAAAU..

5'...AUGAGGCCACAUUCAGCUGCUAU..

3' guguuUgguaAuACACGACGAU

hsa-miR-15b

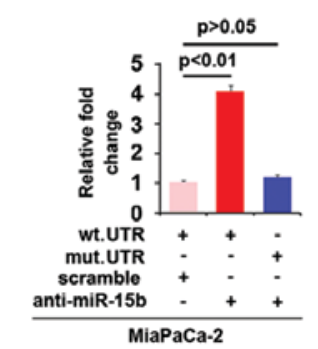

Figure 5. miR-15b suppresses SMURF2 by targeting its 3'-UTR in a pancreatic cancer cell line. (A) Partial heat map of miRNA microarray analysis of MiaPaCa-2 and BxPC-3 cells. n=3. (B) Ven diagram showing the predicted microRNA targeting 3'-UTR of SMURF2 mRNA from databases (TargetSan and PicTar). (C) Schematic of predicted miR-15b-binding sites in the 3'-UTR of SMURF2 mRNA by TargetSan. (D) Real-time PCR for miR-15b in BxPC-3 cells. BxPC-3 cells were infected with pre-miR-15b or control miR (mock). U6 was a loading control. $n=3$. (E) Western blot analysis for SMURF2 protein in BxPC-3 cells infected as indicated. $\beta$-actin was a loading control. $n=3$. (F) Immunofluorescence analyses for BxPC-3 cells transfected with pre-miR-15b and control miR (mock). Left panel shows microscopic images of immunofluorescence staining of one representative experiment (x100 magnification). Right panel shows graphic presentation of mean fluorescence intensities. $\mathrm{n}=3$. (G) RT-PCR for SMURF2 mRNA in BxPC-3 cells infected as indicated. GAPDH was a loading control. $n=3$. (H) Real-time PCR for SMURF2 in BxPC-3 cells infected with pre-miR-15b or control miR (mock). GAPDH was a loading control. $n=3$. (I) Real-time PCR for miR-15b in MiaPaCa-2. MiaPaCa-2 were infected with pre-miR-15b or control miR (mock). U6 was a loading control. $\mathrm{n}=3$. (J) Western blot analysis for SMURF2 protein in MiaPaCa-2 cells infected as indicated. $\beta$-actin was a loading control. $n=3$. (K) RT-PCR for SMURF2 mRNA in MiaPaCa-2 cells infected as indicated. GAPDH was a loading control. $n=3$. (L) Real-time PCR for SMURF2 in MiaPaCa-2 cells infected with pre-miR-15b or control miR (mock). U6 was a loading control. $n=3$. (M) Diagram of SMURF2-3'-UTR-containing reporter constructs. MUT, contains 3-basemutation at the miR-15b-target region, abolishing its binding. (N) Reporter assay, WT-UTR (S-W) but not MUT-UTR (S-M) reporter activity is reduced only in MiaPaCa-2 cells. $\mathrm{n}=3$. (O) Reporter assay, with cotransfection of $500 \mathrm{ng}$ WT- or MUT-reporter and $50 \mathrm{nM}$ control-miR (mock), or pre-miR-15b as indicated. $\mathrm{n}=3$. (P) Reporter assay, with cotransfection of $500 \mathrm{ng}$ WT- or MUT-reporter and $50 \mathrm{nM}$ scramble, or anti-miR-15b as indicated. $\mathrm{n}=3$. 
A

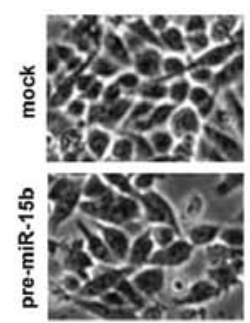

C
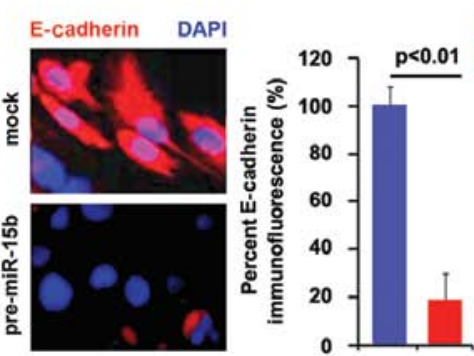

E

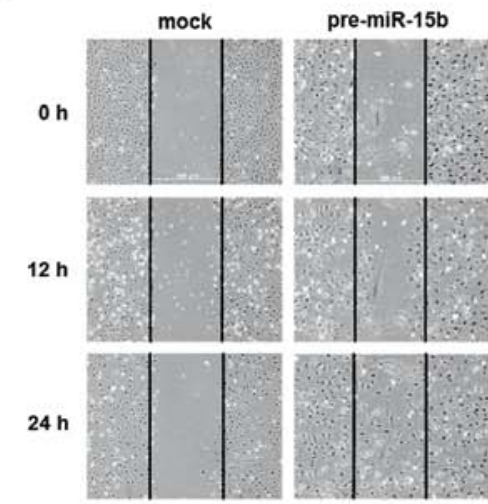

B

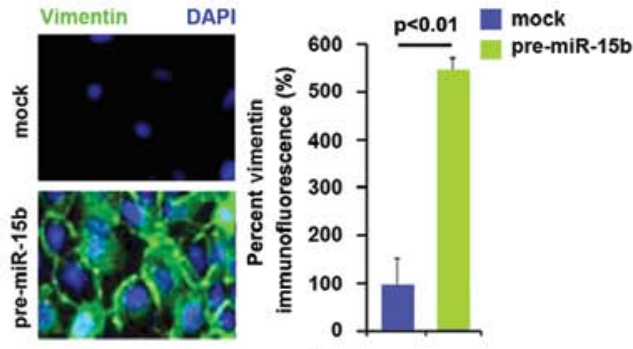

D

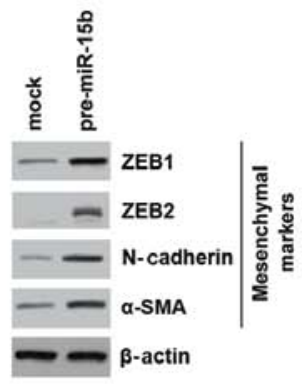

$\mathbf{F}$

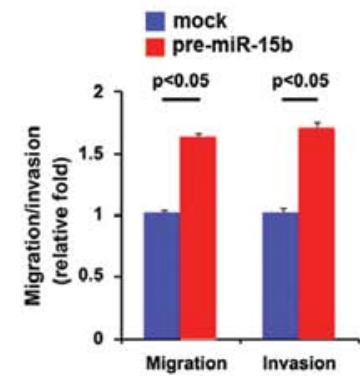

G

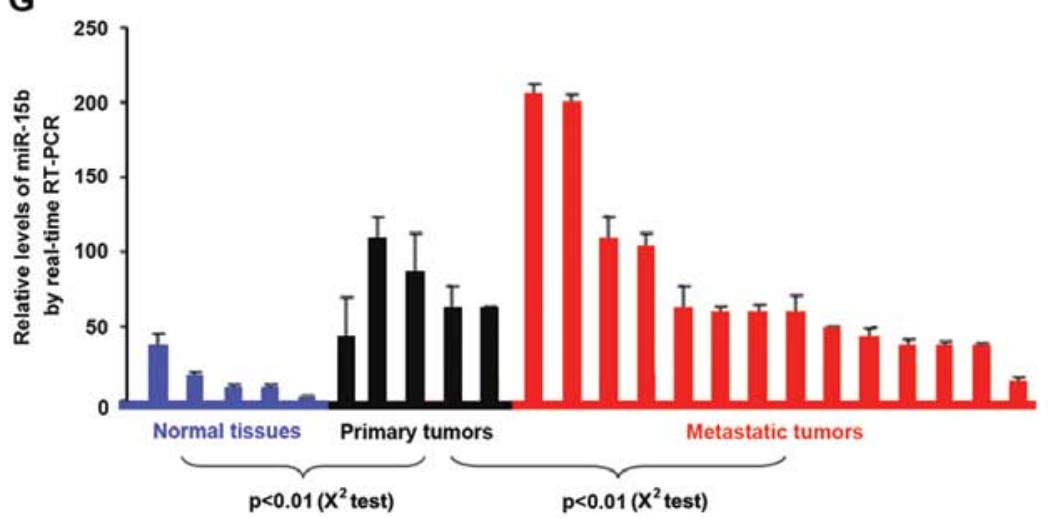

Figure 6. miR-15b promotes EMT in pancreatic cancer cells. (A) BxPC-3 cells were transfected with control miR (mock) or pre-miR-15b. Cells were then photographed. n=3. (B) Immunofluorescence analyses for vimentin in BxPC-3 cells transfected with pre-miR-15b and control miR (mock). Left panel shows microscopic images of immunofluorescence staining of one representative experiment (x100 magnification). Right panel shows graphic presentation of mean fluorescence intensities. n=3. (C) Immunofluorescence analyses for E-cadherin in BxPC-3 cells transfected with pre-miR-15b and control miR (mock). Left panel shows microscopic images of immunofluorescence staining of one representative experiment (x100 magnification). Right panel shows graphic presentation of mean fluorescence intensities. $n=3$. (D) Western blotting for ZEB1, ZEB2, N-cadherin and $\alpha$-SMA protein in BxPC-3 cells transfected with pre-miR-15b and control miR (mock). $\beta$-actin was a loading control. $n=3$. (E) Wound-healing assays for BxPC-3 cells transfected with pre-miR-15b and control miR (mock). The cell layer was photographed. $\mathrm{n}=3$. (F) Invasion and migration assays for BxPC-3 cells transfected with pre-miR-15b and control miR (mock). $\mathrm{n}=3$. (G) Real-time RT-PCR for miR-15b in 19 thyroid cancer tissues from patients with indicated status of metastasis and 5 normal tissues. Error bars indicate SEM of triplicate experiments. $n=24$.

by pre-miR-15b in BxPC-3 cells (Fig. 5D). In an attempt to identify the role of miR-15b in regulating EMT in pancreatic cancer, the cells were transfected with pre-miR-15b. Its overexpression caused changes in BxPC-3 cell morphology (EMT) 
(Fig. 6A). To further verify that the change in cell morphology was caused by EMT, we performed immunoflurescence to analyze expression of epithelial and mesenchymal markers in pancreatic cancer cells. Expression levels of vimentin (mesenchymal markers) and E-cadherin (epithelial marker) were compared in BxPC-3 cells transfected with pre-miR-15b or control miR. The results showed that vimentin was induced by miR-15b overexpression in BxPC-3 cells (Fig. 6B), whereas E-cadherin was evidently repressed by it (Fig. 6C). We also performed western blot analysis and expression was analyzed of other mesenchymal markers in the cells transfected with pre-miR-15b. The results showed that expression of ZEB1, ZEB2, N-cadherin and $\alpha$-SMA were significantly promoted by miR-15b (Fig. 6D). In order to identify whether miR-15b affected migration and invasion, we performed would healing, migration and invasion assay in BxPC-3 cells. The results showed that miR-15b promotes migration (Fig. 6E and F) and invasion (Fig. 6F) in the cells. Having showed that miR-15b was positively associated with invasion and migration in vitro, we performed real-time PCR to analyze whether it was associated with metastasis in pancreatic cancer patients.

The results demonstrated that miR-15b was increased in primary tumors, compared with adjacent normal tissues and it was also higher in metastatic tumors than primary tumors (Fig. 6G). All the results demonstrated that miR-15b promoted EMT and it was positively associated with metastasis in pancreatic cancer.

\section{Disscussion}

Pancreatic cancer ranks fourth in cancer-related mortality, with an overall 5-year survival rate $<1 \%$ and a mean survival time of 4-6 months (38). Late initial diagnosis, chemotherapy and radiation resistance and early metastatic spread accounts for non-satisfactory progress in therapy (39). There is evidence that EMT account for drug resistance, metastasis and late recurrence after years of dormancy (26,40-43). Emerging evidence also suggests that the processes of EMT is regulated by the expression status of tumor suppressive genes, oncogenes and many microRNAs (miRNAs), which are believed to function as key regulators of various biological and pathological processes during tumor development and progression (37,44-46).

Here we present evidence that the expression of SMURF2 protein is downregulated preferentially in pancreatic cancer. The cancer-associated downregulation is consistent with the recent study that suggested the tumor suppressive function of this SMURF2 (47). Low expression of SMURF2 protein was also observed in gemcitabine-resistant cell lines, implying that its abnormal expression was associated with drug-resistance. It is able to promote MET in normal pancreas cells and pancreatic cancer cells. This is also consistent with the report that EMT plays an important role in gemcitabine-resistance (41). TGF- $\beta$ functions as a pro-metastatic factor in human cancer (48). In this study, we demonstrated that SMURF2 inhibited TGF- $\beta$-mediated EMT in normal pancreas cells. This further confirmed that SMURF2 is a tumor suppressive gene. In addition, we found that miR-15b promoted EMT by degrading SMURF2 in pancreatic cancer cells. We found that antagomirs against miR-15b substantially increased SMURF2 levels in the glioblastoma cell lines (unpublished data). Whether anti-
miR-15b could increase SMURF2 levels in pancreatic cancer needs further study.

Collectively, miR-15b-mediated SMURF2 regulation in pancreatic cancer has potential basic and clinical implications. On the one hand, miR-15b could be a powerful oncogene by promoting EMT and regulating relevant tumor suppressor genes in pancreatic cancer, and pharmacological inhibition of miR-15b may represent a promising therapeutic strategy. On another hand, SMURF2 is a tumor suppressor gene and its expression is inhibited by miR-15b in pancreatic cancer. Further studies are clearly required.

\section{References}

1. Hay ED: An overview of epithelio-mesenchymal transformation. Acta Anat (Basel) 154: 8-20, 1995.

2. Pérez-Pomares JM and Muñoz-Chápuli R:Epithelial-mesenchymal transitions: A mesodermal cell strategy for evolutive innovation in Metazoans. Anat Rec 268: 343-351, 2002.

3. Thiery JP and Sleeman JP: Complex networks orchestrate epithelial-mesenchymal transitions. Nat Rev Mol Cell Biol 7: 131-142, 2006.

4. Davies JA: Mesenchyme to epithelium transition during development of the mammalian kidney tubule. Acta Anat (Basel) 156: 187-201, 1996.

5. Nieto MA: The ins and outs of the epithelial to mesenchymal transition in health and disease. Annu Rev Cell Dev Biol 27: 347-376, 2011.

6. Savagner P, Yamada KM and Thiery JP: The zinc-finger protein slug causes desmosome dissociation, an initial and necessary step for growth factor-induced epithelial-mesenchymal transition. J Cell Biol 137: 1403-1419, 1997.

7. Thiery JP: Epithelial-mesenchymal transitions in tumour progression. Nat Rev Cancer 2: 442-454, 2002

8. Cheng GZ, Chan J, Wang Q, Zhang W, Sun CD and Wang LH: Twist transcriptionally up-regulates AKT2 in breast cancer cells leading to increased migration, invasion, and resistance to paclitaxel. Cancer Res 67: 1979-1987, 2007.

9. Comijn J, Berx G, Vermassen P, Verschueren K, van Grunsven L, Bruyneel E, Mareel M, Huylebroeck D and van Roy F: The twohanded $\mathrm{E}$ box binding zinc finger protein SIP1 downregulates E-cadherin and induces invasion. Mol Cell 7: 1267-1278, 2001.

10. Hartwell KA, Muir B, Reinhardt F, Carpenter AE, Sgroi DC and Weinberg RA: The Spemann organizer gene, Goosecoid, promotes tumor metastasis. Proc Natl Acad Sci USA 103: 18969-18974, 2006.

11. Huber MA, Kraut N and Beug H: Molecular requirements for epithelial-mesenchymal transition during tumor progression. Curr Opin Cell Biol 17: 548-558, 2005.

12. Mani SA, Yang J, Brooks M, Schwaninger G, Zhou A, Miura N, Kutok JL, Hartwell K, Richardson AL and Weinberg RA: Mesenchyme Forkhead 1 (FOXC2) plays a key role in metastasis and is associated with aggressive basal-like breast cancers. Proc Natl Acad Sci USA 104: 10069-10074, 2007.

13. Oft M, Akhurst RJ and Balmain A: Metastasis is driven by sequential elevation of H-ras and Smad2 levels. Nat Cell Biol 4: 487-494, 2002.

14. Peinado H, Olmeda D and Cano A: Snail, Zeb and bHLH factors in tumour progression: An alliance against the epithelial phenotype? Nat Rev Cancer 7: 415-428, 2007.

15. Savagner P, Kusewitt DF, Carver EA, Magnino F, Choi C, Gridley $\mathrm{T}$ and Hudson LG: Developmental transcription factor slug is required for effective re-epithelialization by adult keratinocytes. J Cell Physiol 202: 858-866, 2005.

16. Yang J, Mani SA and Weinberg RA: Exploring a new twist on tumor metastasis. Cancer Res 66: 4549-4552, 2006.

17. Chang CJ, Chao CH, Xia W, Yang JY, Xiong Y, Li CW, Yu WH, Rehman SK, Hsu JL, Lee HH, et al: p53 regulates epithelialmesenchymal transition and stem cell properties through modulating miRNAs. Nat Cell Biol 13: 317-323, 2011.

18. Gravdal K, Halvorsen OJ, Haukaas SA and Akslen LA: A switch from $\mathrm{E}$-cadherin to $\mathrm{N}$-cadherin expression indicates epithelial to mesenchymal transition and is of strong and independent importance for the progress of prostate cancer. Clin Cancer Res 13: 7003-7011, 2007 
19. Hader C, Marlier A and Cantley L: Mesenchymal-epithelial transition in epithelial response to injury: The role of Foxc2. Oncogene 29: 1031-1040, 2010.

20. Pasquinelli AE, Reinhart BJ, Slack F, Martindale MQ, Kuroda MI Maller B, Hayward DC, Ball EE, Degnan B, Müller P, et al: Conservation of the sequence and temporal expression of let-7 heterochronic regulatory RNA. Nature 408: 86-89, 2000.

21. Reinhart BJ, Slack FJ, Basson M, Pasquinelli AE, Bettinger JC, Rougvie AE, Horvitz HR and Ruvkun G: The 21-nucleotide let-7 RNA regulates developmental timing in Caenorhabditis elegans. Nature 403: 901-906, 2000.

22. Esteller M: Non-coding RNAs in human disease. Nat Rev Genet 12: 861-874, 2011

23. Esquela-Kerscher A and Slack FJ: Oncomirs - microRNAs with a role in cancer. Nat Rev Cancer 6: 259-269, 2006

24. Garzon R, Calin GA and Croce CM: MicroRNAs in cancer. Annu Rev Med 60: 167-179, 2009.

25. Slack FJ and Weidhaas JB: MicroRNA in cancer prognosis. N Engl J Med 359: 2720-2722, 2008.

26. Li Y, VandenBoom TG II, Kong D, Wang Z, Ali S, Philip PA and Sarkar FH: Upregulation of miR-200 and let-7 by natural agents leads to the reversal of epithelial-to-mesenchymal transition in gemcitabine-resistant pancreatic cancer cells. Cancer Res 69: 6704-6712, 2009.

27. Liao XH, Lu DL, Wang N, Liu LY, Wang Y, Li YQ, Yan TB, Sun XG, Hu P and Zhang TC: Estrogen receptor $\alpha$ mediates proliferation of breast cancer MCF-7 cells via a p21/PCNA/ E2F1-dependent pathway. FEBS J 281: 927-942, 2014

28. Zuo JH, Zhu W, Li MY, Li XH, Yi H, Zeng GQ, Wan XX, He QY, $\mathrm{Li} \mathrm{JH}, \mathrm{Qu} \mathrm{JQ}$, et al: Activation of EGFR promotes squamous carcinoma SCC10A cell migration and invasion via inducing EMT-like phenotype change and MMP-9-mediated degradation of E-cadherin. J Cell Biochem 112: 2508-2517, 2011.

29. Jung H, Lee KP, Park SJ, Park JH, Jang YS, Choi SY, Jung JG, Jo K, Park DY, Yoon JH, et al: TMPRSS4 promotes invasion, migration and metastasis of human tumor cells by facilitating an epithelial-mesenchymal transition. Oncogene 27: 2635-2647, 2008.

30. Christiansen JJ and Rajasekaran AK: Reassessing epithelial to mesenchymal transition as a prerequisite for carcinoma invasion and metastasis. Cancer Res 66: 8319-8326, 2006.

31. Xu J, Lamouille S and Derynck R: TGF-beta-induced epithelial to mesenchymal transition. Cell Res 19: 156-172, 2009.

32. Shimono Y, Zabala M, Cho RW, Lobo N, Dalerba P, Qian D, Diehn M, Liu H, Panula SP, Chiao E, et al: Downregulation of miRNA-200c links breast cancer stem cells with normal stem cells. Cell 138: 592-603, 2009.

33. Lee RC, Feinbaum RL and Ambros V: The $C$ elegans heterochronic gene lin-4 encodes small RNAs with antisense complementarity to lin-14. Cell 75: 843-854, 1993.

34. Meng F, Henson R, Wehbe-Janek H, Ghoshal K, Jacob ST and Patel T: MicroRNA-21 regulates expression of the PTEN tumor suppressor gene in human hepatocellular cancer. Gastroenterology 133: 647-658, 2007.

35. Zhu S, Wu H, Wu F, Nie D, Sheng S and Mo YY: MicroRNA-21 targets tumor suppressor genes in invasion and metastasis. Cell Res 18: 350-359, 2008.
36. Zhu S, Si ML, Wu H and Mo YY: MicroRNA-21 targets the tumor suppressor gene tropomyosin 1 (TPM1). J Biol Chem 282: 14328-14336, 2007.

37. Lee EJ, Gusev Y, Jiang J, Nuovo GJ, Lerner MR, Frankel WL, Morgan DL, Postier RG, Brackett DJ and Schmittgen TD: Expression profiling identifies microRNA signature in pancreatic cancer. Int J Cancer 120: 1046-1054, 2007.

38. Jemal A, Siegel R, Xu J and Ward E: Cancer statistics, 2010. CA Cancer J Clin 60: 277-300, 2010.

39. Wang Z, Li Y, Ahmad A, Banerjee S, Azmi AS, Kong D and Sarkar FH: Pancreatic cancer: Understanding and overcoming chemoresistance. Nat Rev Gastroenterol Hepatol 8: 27-33, 2011.

40. Ellenrieder V, Hendler SF, Boeck W, Seufferlein T, Menke A, Ruhland C, Adler G and Gress TM: Transforming growth factor beta1 treatment leads to an epithelial-mesenchymal transdifferentiation of pancreatic cancer cells requiring extracellular signal-regulated kinase 2 activation. Cancer Res 61: 4222-4228, 2001.

41. Wang Z, Li Y, Kong D, Banerjee S, Ahmad A, Azmi AS, Ali S, Abbruzzese JL, Gallick GE and Sarkar FH: Acquisition of epithelial-mesenchymal transition phenotype of gemcitabineresistant pancreatic cancer cells is linked with activation of the notch signaling pathway. Cancer Res 69: 2400-2407, 2009.

42. Arumugam T, Ramachandran V, Fournier KF, Wang H, Marquis L, Abbruzzese JL, Gallick GE, Logsdon CD, McConkey DJ and Choi W: Epithelial to mesenchymal transition contributes to drug resistance in pancreatic cancer. Cancer Res 69: 5820-5828, 2009.

43. Sarkar FH, Li Y, Wang Z and Kong D: Pancreatic cancer stem cells and EMT in drug resistance and metastasis. Minerva Chir 64: 489-500, 2009.

44. Tucker ON, Dannenberg AJ, Yang EK, Zhang F, Teng L, Daly JM, Soslow RA, Masferrer JL, Woerner BM, Koki AT, et al: Cyclooxygenase-2 expression is up-regulated in human pancreatic cancer. Cancer Res 59: 987-990, 1999.

45. Korc M, Chandrasekar B, Yamanaka Y, Friess H, Buchier M and Beger HG: Overexpression of the epidermal growth factor receptor in human pancreatic cancer is associated with concomitant increases in the levels of epidermal growth factor and transforming growth factor alpha. J Clin Invest 90: 1352-1360, 1992.

46. Boucher MJ, Morisset J, Vachon PH, Reed JC, Lainé J and Rivard N: MEK/ERK signaling pathway regulates the expression of Bcl-2, Bcl-X(L), and Mcl-1 and promotes survival of human pancreatic cancer cells. J Cell Biochem 79: 355-369, 2000.

47. Liu X, Gu X, Sun L, Flowers AB, Rademaker AW, Zhou Y and Kiyokawa H: Downregulation of Smurf2, a tumor-suppressive ubiquitin ligase, in triple-negative breast cancers: Involvement of the RB-microRNA axis. BMC Cancer 14: 57, 2014.

48. Pardali K and Moustakas A: Actions of TGF-beta as tumor suppressor and pro-metastatic factor in human cancer. Biochim Biophys Acta 1775: 21-62, 2007. 